\title{
A New Welding Robot for Intersected Weld of Pipes Used in Large Pressure Vessels
}

\author{
Lianghao Xue, Min Weia and Tao Yang \\ Mechanical and Electrical Engineering College, Shihezi University, Shihezi, 832000, China
}

\begin{abstract}
A self-designed new welding robot to be employed in the intersected weld of large pressure pipes is introduced in this paper. The welding robot can effectively serve as a substitute for a worker who would otherwise face a high labor intensity. The pose model of the welding tool is established to ensure that the welding tool maintains a good pose to make the molten pool run smoothly. The kinematics model of welding robot is established by DenavitHartenberg method, and the trajectory of welding tool is planned and determined. The designed of control system is communicated through PLC and touch screen, based on flexible modular mechanism, the welding tool pose can be adjusted according to the pressure vessel specification and the size of welding seam to ensure the welding quality of welding seam. Finally, the test unit for the designed robot system is set up, and experiments demonstrate that this welding robot could meet the intersected welding requirements of a large pressure vessels environment.
\end{abstract}

\section{Introduction}

Pressure vessels are widely used in petrochemical, coal, electric power and other industries, welding automation technology and welding quality of pressure vessels play a vital role in pressure vessels and even the entire welding industry. With the rapid development of robotics and welding technology, the use of robot in welding industry has grown greatly, and its application has been seen as a typical example in the field of welding automation for large pressure vessels[1].

In the application of large pressure vessel and pipeline engineering, the intersected weld is a typical welding form commonly used. This kind of space curve welding has high labor intensity, and the welding quality is not easy to be guaranteed. Therefore, more and more robot welding is adopted. In recent years, many researchers have made some achievements in the pressure vessels field of welding robot to implement the welding task of pipes intersecting. Shi et al. [2] established the geometric model and the motion model of the automatic welding of the rotating intersecting curve, realized the full process angle of the intersecting welding. Some researchers have designed a right-angle cylindrical coordinate robot with simple structure to achieve easy control and high accuracy[3]. Other researchers have developed a welding robot, which installed on the top of the cylinder, implementing the welding process with the rotation around its axis[4-6]. A synthesized mechanism was chosen as the parallel module of a five degree of freedom hybrid machine tool[7-10]. However, for large intersecting line components, due to their large weight, movement needs greater power, and the cost of tooling is also higher, resulting in more complex and high cost of control system. China and other developing countries currently require flexible and low-cost robot for applications in such environments.

In this paper, firstly, the task requirement and motion analysis of welding robot is addressed, and the design and working principle of welding robot mechanism is described. Secondly, description of the pose of welding tool is addressed, the kinematics model of welding robot was established and the trajectory of welding tool was planned and determined. Finally, the welding robot system was set up and tested, and experiments results reveal that the welding robot is appropriate for practical application.

\section{Task and motion analysis}

In the production of large pressure vessels, such as various high temperature and high pressure reactors and large boilers in petroleum and chemical equipment, the welding of intersecting pipes is one of the most common tasks in practices. The welding part is often made of the main pipe and a branch pipe with different radius, as shown in Figure 1. The weld seam is a saddle-like space curve formed by two cylinders. It is obvious that at different point of the curve, the robot must have different figure to enable its end-effector to point at its aim with good accuracy and pose. So, the robot must have a good dexterity during the whole welding process. In real practice, the continuous operation is required in welding process, so it is necessary to ensure that the robots do not interfere with each other.

\footnotetext{
a Corresponding author: Min Wei, 1448237074@qq.com
} 
The motion of the robot during the welding process can be divided into four parts. The first motion is the rotation of the robot around the central axis of the branch. The robot is usually installed on the branch pipe and concentric with its axis. Obviously, this motion only needs one degree of freedom. The second motion is that the robot adjusts the lateral movement according to the diameter of the branch pipe, this motion requires one degree of freedom. The third movement is that the robot adjusts the longitudinal movement according to the height of the branch pipe, this motion requires one degree of freedom. The fourth motion is to adjust the pose of the welding tool in time according to the real-time position of the weld at work to ensure the welding quality of the weld, this motion also requires one degree of freedom. In all, the motion of the robot requires 4 degree of freedom to complete the welding work.

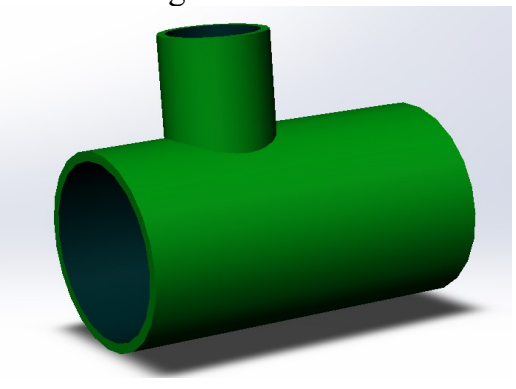

Figure 1. Intersected pipes

\section{Design and working principle of welding robot mechanism}

According to the characteristics of the saddle weld curve and the welding process requirements of the saddle weld, the welding robot mechanism mainly consists of cross suspension moving mechanism, lifting and positioning mechanism, body rotating mechanism and welding gun adjusting mechanism, etc., as shown in Figure 2. Through the coordinated movement among the various mechanisms to achieve large pressure vessel saddleshaped welding operations.

\subsection{Cross suspension moving mechanism}

Cross hanging mobile mechanism driven by motor, ball screw, linear guide in mutual cooperation, realize the robot on the lifting beam platform of radial and lateral movement, and the welding robot for heavy pressure vessel saddle saddle-shaped seam welding operation before taking over and coordinates of center positioning, as shown in Figure 2.

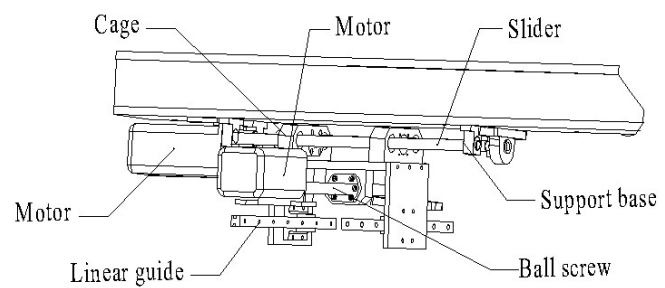

Figure 2. Cross suspension moving mechanism.

\subsection{Lifting and positioning mechanism}

The lifting and positioning mechanism drives the rotary shaft of the chuck to rotate through the motor, so as to realize the connection rotation of the branch pipe clamped on the pneumatic three-jaw chuck. The connection between the branch pipe and the saddle groove is realized with the cooperation of the pressing action of the thin cylinder. As shown in Figure 3.

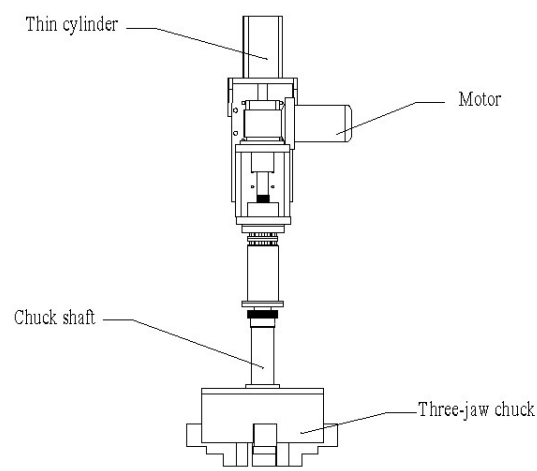

Figure 3. Lifting and positioning mechanism.

\subsection{Rotating mechanism}

The rotating mechanism of the machine body realizes the rotating motion of the welding gun in the saddle welding process and the radial motion in the $\mathrm{x}$ and $\mathrm{y}$ axis plane through the motor driving small belt wheel and the motor driving ball screw. As shown in Figure 4.

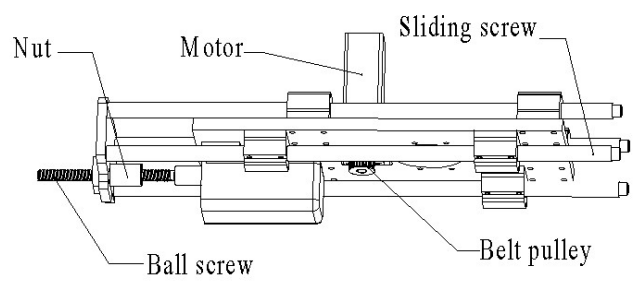

Figure 4. Rotating mechanism.

\subsection{Welding gun adjustment mechanism}

The welding gun adjusting mechanism is driven by the motor and driven by the belt wheel, and it can realize the linear interpolation motion of the welding gun in the zaxis direction with the cooperation motion of the linear guide rail. Through the motor drive shaft movement to achieve the welding gun position Angle adjustment. As shown in Figure 5.

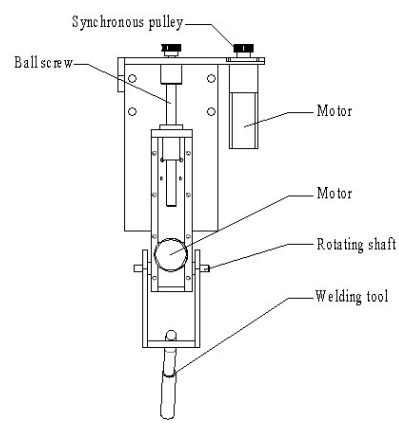

Figure 5. Welding gun adjustment mechanism. 
The welding robot realizes the welding of the saddle weld of large pressure vessel through the coordinated movement of each mechanism. The specific performance parameters are shown in table 1 .

Table 1. Performance parameters of welding robot.

\begin{tabular}{cc}
\hline Parameter & Value \\
\hline Radius of cylinder & $700-2000 \mathrm{~mm}$ \\
Radius of branch pipe & $25-250 \mathrm{~mm}$ \\
Height of branch pipe & $100-400 \mathrm{~mm}$ \\
Pose angle & $15^{\circ}-60^{\circ}$ \\
Rotary speed & $0-4 \mathrm{r} / \mathrm{min}$ \\
Welding type & GMAW \\
\hline
\end{tabular}

\section{Welding robot kinematics model and trajectory planning}

\subsection{Forward kinematics analysis of welding robot}

In this study, coordinate systems between links are established based on classical D-H method[11,12], and a kinematics model of a saddle weld welding robot with joint angle as a variable is proposed, as shown in Figure 6. The coordinate systems consists of four parameters, the link offset" $d$ ", joint angle" $\theta$ ", link length" $a$ "and twist angle" $\alpha$ ", where the joint angle " $\theta$ "is the joint variable, and the other three parameters are constants remain unchanged. Link parameter values are given in table 2 .

Table 2. Welding robot links parameters.

\begin{tabular}{ccccc}
\hline$i$ & $a_{i}$ & $\alpha_{i}$ & $d_{i}$ & $\theta_{i}$ \\
\hline 1 & 0 & $-90^{\circ}$ & $d_{1}$ & $\theta_{1}$ \\
2 & 0 & $-90^{\circ}$ & $d_{2}$ & 0 \\
3 & 0 & $-90^{\circ}$ & $d_{3}$ & $-90^{\circ}$ \\
4 & $a_{4}$ & 0 & 0 & $\theta_{4}$ \\
\hline
\end{tabular}

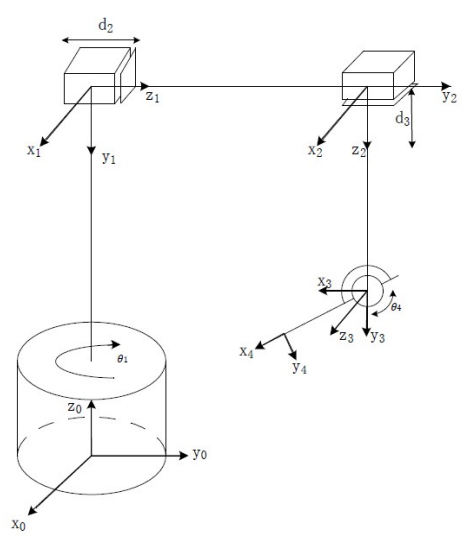

Figure 6. Welding robot coordinate system of the D-H.

The kinematics problem describes the relationship between the joints of the saddle-shaped weld welding robot and the position and attitude of the end of the welding torch. That is, the position of the welding gun end coordinate system relative to the base coordinate system is solved under the condition of the geometrical parameters of the bar and the joint displacement.

According to the $\mathrm{D}-\mathrm{H}$ convention, we use the homogeneous transformation matrix to represent the position and attitude of the first coordinate system, which is written as:

$$
\mathbf{A}_{\mathbf{i}}=\left[\begin{array}{cccc}
\cos \theta_{i} & -\cos \alpha_{i} \sin \theta_{i} & \sin \alpha_{i} \sin \theta_{i} & a_{i} \cos \theta_{i} \\
\sin \theta_{i} & \cos \alpha_{i} \cos \theta_{i} & -\sin \alpha_{i} \cos \theta_{i} & a_{i} \sin \theta_{i} \\
0 & \sin \alpha_{i} & \cos \alpha_{i} & d_{i} \\
0 & 0 & 0 & 1
\end{array}\right]
$$

Bring the parameters of each link into the above equation to get:

$$
\begin{gathered}
\mathbf{A}_{\mathbf{1}}=\left[\begin{array}{cccc}
\cos \theta_{1} & 0 & -\sin \theta_{1} & 0 \\
\sin \theta_{1} & 0 & \cos \theta_{1} & 0 \\
0 & -1 & 0 & d_{1} \\
0 & 0 & 0 & 1
\end{array}\right] \\
\mathbf{A}_{2}=\left[\begin{array}{cccc}
1 & 0 & 0 & 0 \\
0 & 0 & 1 & 0 \\
0 & -1 & 0 & d_{2} \\
0 & 0 & 0 & 1
\end{array}\right] \\
\mathbf{A}_{\mathbf{3}}=\left[\begin{array}{cccc}
0 & 0 & 1 & 0 \\
-1 & 0 & 0 & 0 \\
0 & -1 & 0 & d_{3} \\
0 & 0 & 0 & 1
\end{array}\right] \\
\mathbf{A}_{\mathbf{4}}=\left[\begin{array}{cccc}
\cos \theta_{4} & -\sin \theta_{4} & 0 & a_{4} \cos \theta_{4} \\
\sin \theta_{4} & \cos \theta_{4} & 0 & a_{4} \sin \theta_{4} \\
0 & 0 & 1 & 0 \\
0 & 0 & 0 & 1
\end{array}\right]
\end{gathered}
$$

Therefore, the homogeneous transformation matrix $\mathbf{T}$ of the $i$-coordinate system relative to the base coordinate pose can be given by

$$
\mathbf{T}_{\mathbf{i}}^{\mathbf{0}}=\mathbf{A}_{1} \mathbf{A}_{2} \ldots \mathbf{A}_{\mathbf{i}}
$$

In particular, when $i=4$, it determines the position and attitude of the welding robot torch tip relative to the base coordinate system, and the $\mathbf{T}$ matrix can be expressed as:

$$
\mathbf{T}=\mathbf{T}_{\mathbf{4}}^{\mathbf{0}}=\mathbf{A}_{\mathbf{1}} \mathbf{A}_{\mathbf{2}} \mathbf{A}_{3} \mathbf{A}_{4}
$$

And

$$
\mathbf{T}_{\mathbf{4}}^{\mathbf{0}}=\left[\begin{array}{cccc}
-\sin \theta_{1} \cos \theta_{4} & \sin \theta_{1} \sin \theta_{4} & \cos \theta_{1} & p_{x} \\
\cos \theta_{1} \cos \theta_{4} & -\cos \theta_{1} \sin \theta_{4} & \sin \theta_{1} & p_{y} \\
\sin \theta_{4} & \cos \theta_{4} & 0 & p_{z} \\
0 & 0 & 0 & 1
\end{array}\right]
$$

Among

$$
\begin{aligned}
& p_{x}=-a_{4} \sin \theta_{1} \cos \theta_{4}-d_{2} \sin \theta_{1} \\
& p_{y}=a_{4} \cos \theta_{1} \cos \theta_{4}+d_{2} \cos \theta_{1} \\
& p_{z}=a_{4} \sin \theta_{4}+d_{1}-d_{3}
\end{aligned}
$$

It can be concluded from the matrix $\mathbf{T}_{\mathbf{4}}^{\mathbf{0}}$ that the first three elements in the last column are the components of the $\mathrm{X}, \mathrm{Y}$ and $\mathrm{Z}$ directions of the origin $\mathrm{O}_{4}$ of the torch end coordinate system in the base coordinate system, that is, the coordinates of the end of the torch in the base coordinate system. The azimuth angle of the torch end 
coordinate system relative to the base coordinate system is given by the rotating portion of the matrix.

\subsection{Inverse kinematics analysis of welding robot}

The inverse kinematics problem is to find the variable parameters of the corresponding joints under the condition of the position and attitude of the welding robot gun, and to satisfy the pose motion of the welding gun terminal under the drive of the control motor of each joint. For a saddle-shaped welding robot with four degrees of freedom(as shown above), the parameters $a_{i}, \alpha_{i}$, and $d_{i}$ of matrix $\mathbf{T}_{\mathbf{4}}^{\mathbf{0}}$ and each link are known, and the values of joint variables $\theta_{1}, \theta_{2}, \theta_{3}$ and $\theta_{4}$ need to be solved, among which $\theta_{2}=d_{2}$ and $\theta_{3}=d_{3}$.

By using analytic method, the two ends of the motion Eq. 16 are respectively multiplied by the inverse matrix of A matrix, which can be obtained

$$
\mathbf{A}_{1}^{-1} \mathbf{T}_{4}^{0}=\mathbf{A}_{1}^{-1} \mathbf{A}_{1} \mathbf{A}_{2} \mathbf{A}_{3} \mathbf{A}_{4}=\mathbf{A}_{2} \mathbf{A}_{3} \mathbf{A}_{4}
$$

By expanding the two ends of the equation 10 of motion and making the corresponding terms at both ends equal, $\theta_{1}$ can be obtained. Similarly, the values of $\theta_{2}, \theta_{3}$ and $\theta_{4}$ can be obtained sequentially by using this method.

\subsection{Welding robot trajectory planning}

Robot track refers to the displacement, speed and acceleration of the robot in the process of motion. According to the task requirements, two different schemes can be used to calculate the expected trajectory and complete the trajectory planning. If you only need to satisfy the start and end states and do not pay attention to the path along the way, you can use the point to point trajectory planning; If the starting and ending states of the robot are specified, and the robot is required to move along a specific path and pass through several intermediate points between two points, the continuous path trajectory planning is required.

The saddle-shaped weld welding robot with four degrees of freedom designed in this paper requires the end of the welding torch to move along a specific path, and continuously passes through each intermediate point to complete the welding task of the saddle-shaped weld. Therefore, the trajectory planning of continuous path motion is adopted. Program. Using the cubic polynomial interpolation method, the joint angle function is used to calculate the interpolation of the saddle weld welding robot.

In the welding process of the saddle welding robot, as the desired velocity of the joint angle $\theta_{0}$ of the starting point and any intermediate point of each joint is known, the joint angle $\theta_{f}$ of the termination point can be obtained by inverse kinematics solution, and the end of the welding gun is required to pass through each intermediate point continuously along the specific path of the saddle weld. Therefore, $\theta(t)$ the description of the motion trajectory can be expressed by a smooth interpolation function of the joint Angle of the starting point and the ending point. At this point, the four constraint conditions met are:

$$
\left\{\begin{array}{l}
\theta\left(t_{0}\right)=\theta_{0} \\
\theta\left(\mathrm{t}_{f}\right)=\theta_{f} \\
\dot{\theta}\left(t_{0}\right)=\theta_{0} \\
\dot{\theta}\left(\mathrm{t}_{f}\right)=\dot{\theta}_{f}
\end{array}\right.
$$

According to the above four constraints, four linear equations with four unknown quantities can be determined, in the form of:

$$
\left\{\begin{array}{l}
\theta\left(t_{0}\right)=a_{0}+a_{1} t_{0}+a_{2} t_{0}{ }^{2}+a_{3} t_{0}{ }^{3} \\
\dot{\theta}\left(t_{0}\right)=a_{1}+2 a_{2} t_{0}+3 a_{3} t_{0}{ }^{2} \\
\theta\left(t_{f}\right)=a_{0}+a_{1} t_{f}+a_{2} t_{f}{ }^{2}+a_{3} t_{f}{ }^{3} \\
\dot{\theta}\left(t_{f}\right)=a_{1}+2 a_{2} t_{f}+3 a_{3} t_{f}{ }^{2}
\end{array}\right.
$$

After calculation, the determinant of the matrix equation coefficient matrix is equal to $\left(t_{f}-t_{0}\right)^{4}$, so there is always A unique solution to the motion track of the saddle weld in non-zero time. After solving the above equations, the following equation can be obtained:

$$
\left\{\begin{array}{l}
a_{0}=\theta_{0} \\
a_{1}=\dot{\theta}_{0} \\
a_{2}=\frac{3\left(\theta_{f}-\theta_{0}\right)}{t_{f}^{2}} \\
a_{3}=-\frac{2\left(\theta_{f}-\theta_{0}\right)}{t_{f}^{3}}
\end{array}\right.
$$

\section{Establishment of welding robot Three- Dimensional Model in MATLA}

According to the parameters in table1, functions "link" and "robot" in MATLAB Robotic Toolbox are used to establish the three-dimensional model of welding robot. The programs of the built robot model are as follows:

$>>$ L1 $=\operatorname{link}([-\mathrm{pi} / 2,0, \mathrm{pi}, 0,0]$,'standard');

$>>$ L2 $=\operatorname{link}([-\mathrm{pi} / 2,0,0,-0.548,1]$,'standard');

$>$ L 3 $=\operatorname{link}([-\mathrm{pi} / 2,0,-$ pi/2,0.342, 1],'standard');

$>>$ L4 $=\operatorname{link}([0,0.158,-$ pi $/ 4,0,0]$, 'standard');

$>\mathrm{r}=\operatorname{robot}(\{\mathrm{L} 1, \mathrm{~L} 2, \mathrm{~L} 3, \mathrm{~L} 4\})$;

$>>$ r.name $=$ 'welding robot';

$>$ drivebot(r);

The above programs are run in MATLAB, and the initial state of the welding robot three-dimensional model is built as shown in Figure 7.

The end position and orientation matrix can be obtained by function "jtraj", and thus the end position coordinates are gotten. Furthermore, the robot end-effector trajectory is simulated. Here still use the initial position "qz" and end position "qr", add the following programs after programs the welding robot three-dimensional mode:

$>\mathrm{t}=[0: 0.01: 10]$;

$>$ qA $=\left[\begin{array}{llll}0 & 0 & 0 & -\mathrm{pi} / 4\end{array}\right]$

$>>\mathrm{qAB}=[2 *$ pi $00-$ pi/4];

$>>\mathrm{q}=\mathrm{j} \operatorname{traj}(\mathrm{qA}, \mathrm{qAB}, \mathrm{t})$;

$>>\mathrm{T}=$ fkine $(\mathrm{r}, \mathrm{q})$; 
$>>\mathrm{x}=$ squeeze $(\mathrm{T}(1,3,:))$;

$>>\mathrm{y}=$ squeeze $(\mathrm{T}(1,2,:))$;

$>>\mathrm{z}=$ squeeze $(\mathrm{T}(1,1,:))$

$>>$ figure;plot3(x,y,z);

Run the above programs to obtain the welding robot end trajectory curve of $\mathrm{x}, \mathrm{y}$, and $\mathrm{z}$-axis directions, the end space trajectory curve as shown in Figure 8.

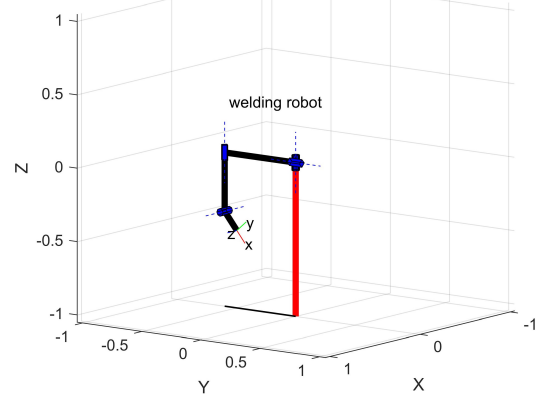

Figure 7. Welding robot three-dimensional model.

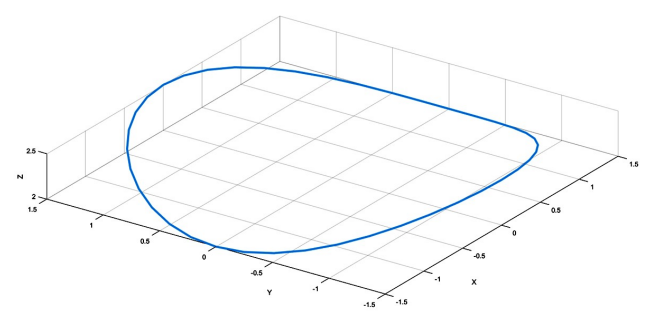

Figure 8. Welding robot end space trajectory curve.

\section{Robot control system and experiments}

The saddle welding robot control system is composed of the upper machine touch screen, lower machine PLC controller, cantilever control box, servo motor, servo driver, ac motor, governor and so on. The overall structure of the control system as shown in Figure 9.

The servo driver is used to drive the servo motor, which requires the PLC to produce high frequency pulse. As the main controller, PLC uses its multichannel pulse output function to output the pulse to the driver, the motor transforms the received pulse signal into corresponding angular displacement and angular velocity control the motor of each actuator, realize multi-axis linkage, and complete the welding of saddle weld.

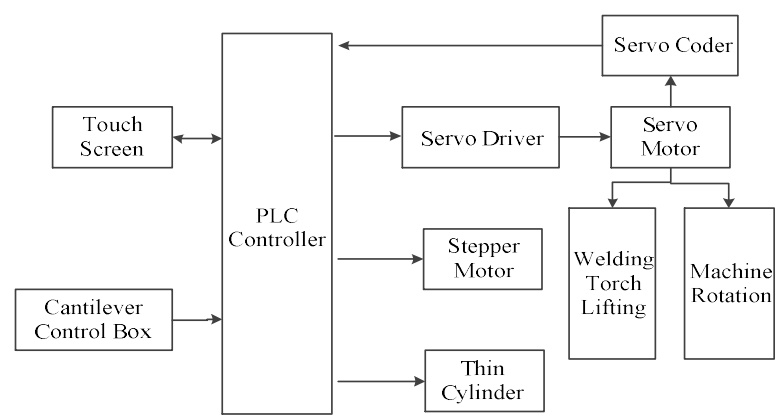

Figure 9. Structure of the control system.

Before the welding work, firstly, the prepared saddleshaped weld interpolation program is uploaded to the PLC, and then the corresponding welding process parameters are input in the human-machine interface of the touch screen, and the touch screen of the upper computer extracts the welding gun in the space by algorithm analysis. The running track in the middle, and calculate the motion pulse and speed of each axis according to the space curve, the various motion parameters are stored in advance in the designated storage area of the PLC, and save them in the form of documents. When the control program starts running, the PLC enters the scan cycle, and the PLC performs the operation in the order of the motion control program edited by the user. When it detects that the pulse output condition is met or the drive motor command is encountered, the pulse and speed are extracted to the predetermined address.

According to the welding process requirements, select the appropriate pressure vessel to carry out the welding test of saddle weld. During the experiment, the three-jaw pneumatic chuck is positioned by clamping the pipe and the saddle weld on the barrel. After positioning is completed, the positioning before welding can be completed by pressing the chuck clamping control button. After that, press the automatic welding button, the welding gun will automatically go around the welding track of the saddle weld without arc starting, verify the accuracy of the welding track of the saddle weld, and after verification, press the welding preparation and automatic welding button, the welding gun will officially enter the welding work, and carry out welding according to the track just walked.The welding picture as shown in Figure 10 .

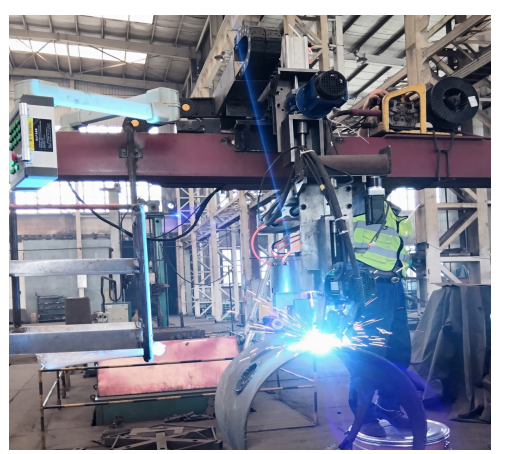

Figure 10. Welding picture.

The welding experimental parameters selected in this experiment are shown in the table 3 . The photograph of the saddle seam surface are shown in Figure 11. The field operation of welding robot shows that the welding gun can weld the welding seam according to the preset welding track, the control scheme and performance of the whole robot system are acceptable and appropriate for practical applications.

Table 3. Welding experiment parameters.

\begin{tabular}{cccc}
\hline Parameter & Value & Parameter & \multicolumn{1}{c}{ Value } \\
\hline Radius of cylinder & $600 \mathrm{~mm}$ & Welding speed & $80 \mathrm{~mm} / \mathrm{min}$ \\
Radius of branch pipe & $150 \mathrm{~mm}$ & Arc voltage & $25.3 \mathrm{~V}$ \\
Welding type & GMAW & Wire diameter & $1.2 \mathrm{~mm}$ \\
Welding current & $230 \mathrm{~A}$ & Pose angle & $45^{\circ}$ \\
\hline
\end{tabular}




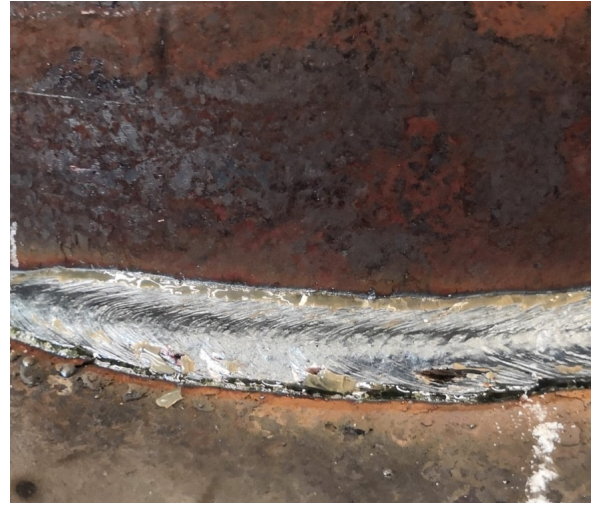

Figure 11. Photograph of the saddle seam surface.

\section{Conclusion}

A new welding robot, to be employed in large pressure vessels, for intersected weld was designed. The mechanical structure of the welding robot, mainly consisting of cross suspension moving mechanism, lifting and positioning mechanism, body rotating mechanism and welding gun adjusting mechanism, has the advantages of accurate positioning and convenient mounting, and can adjust the size of the cross suspension moving mechanism to adapt to different practical requirements in intersected weld of pipes. Description of the pose of welding tool during welding and the kinematics model of the welding robot with the joint angle as the variable was established. Using the MATLAB Robotics Toolbox to simulate the welding robot kinematics. Finally, the welding robot system was set up and tested, and experiments results reveal that the welding robot is appropriate for practical application. The application of such flexible and low-cost robot not only improve the efficiency and weld consistency, but also protects the operators from high labour intensity.

\section{References}

1. Z. Xiaodong, W. Yuanyu, H. Pengfei. Machine Design Research, 24, 21-24(2008)

2. S. Lei, T. Xincheng. Transactions of the China Welding Institution, 36, 5-8(2015)

3. L. Xiaohui, W. Su. Journal of Beijing University of Aeronautics and Astronautics, 34, 964-968(2008)

4. R. Fushen, C .hujun, G. Xinyong. Transactions of the China Welding Institution, 30, 59-62(2009)

5. H. Liguo, Baron L. Industrial Robot, 35, 456464(2008)

6. W. Kundong, Y. Guozheng, Y. Bo. Advanced Robotics, 23, 1579-1599(2009)

7. M. Okada, H. Kozuka, H. Tachiya, T. Iwasaki, and Y. Ya-mashita. Int. J. of Automation Technology, 243252(2014)

8. D. pisla, A. Szilaghyi, C. Vaida, and N. Plitea Robotics and Computer-Integrated Manufacturing, 463-474 (2013)

9. P. Ben-Tzvi. Journal of Field Robotics, 250267(2013)

10. F. Xie, X. J.Liu, Z. You and J. Wang. Robotics and Computer-Integrated Manufacturing, 1-10(2014)

11. S. Wei, Z. Hua, Q. Jinghui. Electric Machines and Control, 16, 78 -86(2012)

12. $\mathrm{C}$. $\mathrm{Yu}, \mathrm{X}$. Fayin. Journal of Chongqing University of Arts and Sciences, 35, 79 -83(2016) 